\title{
Positive solutions of BVPs for infinite difference equations with one-dimensional p-Laplacian
}

\author{
Jianye Xia and Yuji Liu
}




\title{
POSITIVE SOLUTIONS OF BVPS FOR INFINITE DIFFERENCE EQUATIONS WITH ONE-DIMENSIONAL $p$-LAPLACIAN
}

\author{
JIANYE XIA AND YUJI LIU
}

Received 4 May, 2011

\begin{abstract}
Sufficient conditions guaranteeing the existence of three positive solutions of the multipoint boundary value problem for the infinite difference equation

$$
\left\{\begin{array}{l}
\Delta[p(n) \phi(\Delta x(n))]+f(n, x(n), \Delta x(n))=0, n \in N_{0}, \\
x(0)-\sum_{n=1}^{\infty} \alpha_{n} x(n)=0, \\
\lim _{n \rightarrow+\infty} \frac{x(n)}{1+\sum_{s=0}^{n-1} \frac{1}{\phi^{-1}(p(s))}}-\sum_{n=1}^{\infty} \beta_{n} x(n)=0,
\end{array}\right.
$$

are established using a fixed point theorem. It is the purpose of this paper to show that this approach of obtaining positive solutions of BVPs by using multi-fixed-point theorems can be extended to infinite difference equations containing the nonlinear operator $\Delta[p \phi(\Delta x)]$. The possible solutions of this BVP are not concave if $p(n) \not \equiv$ constant.
\end{abstract}

2000 Mathematics Subject Classification: 34B10; 34B15; 35B10

Keywords: one-dimension $p$-Laplacian infinite difference equation, multi-point boundary value problem, positive solution, fixed point theorem

\section{INTRODUCTION}

In recent years, there have been many papers interested in proving the existence of positive solutions of the boundary value problems (BVPs for short) of the finite difference equations since these BVPs have extensive applications, see the papers $[1,5-9,11-15,18,19],[10]$ and the references therein.

Recently, the authors $[2-4,16,17]$ studied the existence of solutions of the boundary value problems for infinite difference equations. In [17], the existence of multiple positive solutions of boundary value problems for the second-order discrete equations

$$
\left\{\begin{array}{l}
\Delta^{2} x(n-1)-p \Delta x(n-1)-q x(n-1)+f(n, x(n))=0, n \in N, \\
\alpha x(0)-\beta \Delta x(0)=0, \\
\lim _{n \rightarrow+\infty} x(n)=0,
\end{array}\right.
$$

Supported by Natural Science Foundation of Hunan province, P.R.China(No:06JJ5008) and Natural Science Foundation of Guangdong province(No:7004569). 
was investigated by using the cone compression and expansion and fixed point theorems in Fréchet spaces, where $\alpha>0, \beta>0, p>0, q>0$ and $f$ is a continuous function.

In paper [2], the existence of solutions of a class of the infinite time scale boundary value problems was considered. It is easy to see that the results of [2] can be applied to the following BVP for the infinite difference equation

$$
\left\{\begin{array}{l}
\Delta^{2} x(n)+f(n, x(n))=0, n \in N_{0}, \\
x(0)=0, \\
x(n) \text { is bounded. }
\end{array}\right.
$$

The methods are based upon a growth argument and the upper and lower solutions methods.

In [12] the existence of at least three positive solutions for the following BVP of the finite difference equations

$$
\left\{\begin{array}{l}
\Delta[\phi(\Delta x(n))]+f(n, x(n), \Delta x(n))=0, n \in[0, N] \\
x(0)-\sum_{i=1}^{m} \alpha_{i} x\left(n_{i}\right)=0 \\
x(N+2)-\sum_{i=1}^{m} \beta_{i} x\left(n_{i}\right)=0
\end{array}\right.
$$

was proved under some assumptions.

Motivated by above mentioned two papers, the purpose of this paper is to investigate the multi-point boundary value problem of the second order infinite $p$-Laplacian difference equation

$$
\left\{\begin{array}{l}
\Delta[p(n) \phi(\Delta x(n))]+f(n, x(n), \Delta x(n))=0, n \in N_{0} \\
x(0)-\sum_{n=1}^{\infty} \alpha_{n} x(n)=0, \\
\lim _{n \rightarrow+\infty} \frac{x(n)}{1+\sum_{s=0}^{n-1} \frac{1}{\phi^{-1}(p(s))}}-\sum_{n=1}^{\infty} \beta_{n} x(n)=0
\end{array}\right.
$$

where $N_{0}$ denotes the set of all nonnegative integers, $p(n)>0, \alpha_{n} \geq 0, \beta_{n} \geq 0$ for all $n \in N_{0}$ and satisfy

$$
\begin{gathered}
\sum_{n=1}^{\infty} \alpha_{n}<1, \\
\sum_{n \in N} \alpha_{n} \sum_{i=0}^{n-1} \frac{1}{\phi^{-1}(p(i))}<\infty
\end{gathered}
$$

and

$$
\frac{\sum_{n=1}^{\infty} \beta_{n}}{1-\sum_{n=1}^{\infty} \alpha_{n}} \sum_{n=1}^{\infty} \alpha_{n} \sum_{j=0}^{n-1} \phi^{-1}\left(\frac{1}{p(j)}\right)+\sum_{n=1}^{\infty} \beta_{n} \sum_{j=0}^{n-1} \phi^{-1}\left(\frac{1}{p(j)}\right)<1,
$$

and

$$
\sum_{s=0}^{\infty} \frac{1}{\phi^{-1}(p(s))}=\infty
$$


$f$ is a Caratheodory function, i.e., $f$ satisfies that for each $r>0$ there exist a real number sequence $\left\{\phi_{r}(n)\right\}$ with $\sum_{n=0}^{\infty} \phi_{r}(n)<\infty$ such that

$$
f\left(n,\left(1+\sum_{s=0}^{n-1} \frac{1}{\phi^{-1}(p(s))}\right) x, \frac{1}{\phi^{-1}(p(n))} y\right) \leq \phi_{r}(n), n \in N_{0},|x| \leq r,|y| \leq r,
$$

$\phi$ is defined by $\phi(x)=|x|^{p-2} x$ with $p>1$, its inverse function is denoted by $\phi^{-1}(x)=|x|^{q-2} x$ with $1 / p+1 / q=1, \Delta x(n)=x(n+1)-x(n)$. We establish sufficient conditions for the existence of at least three positive solutions of $\mathrm{BVP}(3)$.

It is easy to see that the positive solutions of BVP(1) are bounded, the positive solutions of BVP(3) may be unbounded since 1.4 holds. The results in this article generalize the theorems in [12] to the infinite case and the fixed point theorem used is different from those used in $[3,12,17]$. The most interesting part in this article is the construction of the nonlinear operator and the cone, this method is not found in known papers.

The remainder of this paper is organized as follows: in Section 2, we first give some lemmas, then the main result (Theorem 1) and its proof are presented. An example is given in Section 3 to illustrate the main result.

\section{MAIN RESUlTS}

In this section, we first present some background definitions in Banach spaces and state three important fixed point theorems and lemmas. Then the main result is given and proved.

Definition 1. Let $X$ be a real Banach space. The nonempty convex closed subset $P$ of $X$ is called a cone in $X$ if $a x \in P$ for all $x \in P$ and $a \geq 0, x \in X$ and $-x \in X$ imply $x=0$.

Definition 2. A map $\psi: P \rightarrow[0,+\infty)$ is a nonnegative continuous concave or convex functional map provided $\psi$ is nonnegative, continuous and satisfies $\psi(t x+$ $(1-t) y) \geq t \psi(x)+(1-t) \psi(y)$, or $\psi(t x+(1-t) y) \leq t \psi(x)+(1-t) \psi(y)$, for all $x, y \in P$ and $t \in[0,1]$.

Definition 3. An operator $T ; X \rightarrow X$ is completely continuous if it is continuous and maps bounded sets into pre-compact sets.

Definition 4. Let $a, b, c, d, h>0$ be positive constants, $\alpha, \psi$ be two nonnegative continuous concave functionals on the cone $P, \gamma, \beta, \theta$ be three nonnegative continuous convex functionals on the cone $P$. Define the convex sets as follows:

$$
\begin{aligned}
& P_{c}=\{x \in P:\|x\|<c\}, \\
& P(\gamma, \alpha ; a, c)=\{x \in P: \alpha(x) \geq a, \gamma(x) \leq c\}, \\
& P(\gamma, \theta, \alpha ; a, b, c)=\{x \in P: \alpha(x) \geq a, \theta(x) \leq b, \gamma(x) \leq c\}, \\
& Q(\gamma, \beta ;, d, c)=\{x \in P: \beta(x) \leq d, \gamma(x) \leq c\},
\end{aligned}
$$




$$
Q(\gamma, \beta, \psi ; h, d, c)=\{x \in P: \psi(x) \geq h, \beta(x) \leq d, \gamma(x) \leq c\} .
$$

Lemma 1 ([7]). Let $X$ be a real Banach space, $P$ be a cone in $X, \alpha, \psi$ be two nonnegative continuous concave functionals on the cone $P, \gamma, \beta, \theta$ be three nonnegative continuous convex functionals on the cone $P$. There exist constant $M>0$ such that

$$
\alpha(x) \leq \beta(x),\|x\| \leq M \gamma(x) \text { for all } x \in P .
$$

Furthermore, Suppose that $h, d, a, b, c>0$ are constants with $d<a$. Let $T: \overline{P_{c}} \rightarrow$ $\overline{P_{c}}$ be a completely continuous operator. If

(C1) $\{y \in P(\gamma, \theta, \alpha ; a, b, c) \mid \alpha(x)>a\} \neq \varnothing$ and

$$
\alpha(T x)>\text { a for every } x \in P(\gamma, \theta, \alpha ; a, b, c) ;
$$

(C2) $\{y \in Q(\gamma, \theta, \psi ; h, d, c) \mid \beta(x)<d\} \neq \varnothing$ and

$$
\beta(T x)<d \text { for every } x \in Q(\gamma, \theta, \psi ; h, d, c) ;
$$

(C3) $\alpha(T y)>a$ for $y \in P(\gamma, \alpha ; a, c)$ with $\theta(T y)>b$;

(C4) $\beta(T x)<d$ for each $x \in Q(\gamma, \beta ;, d, c)$ with $\psi(T x)<h$,

then $T$ has at least three fixed points $y_{1}, y_{2}$ and $y_{3}$ such that

$$
\beta\left(y_{1}\right)<d, \alpha\left(y_{2}\right)>a, \beta\left(y_{3}\right)>d, \alpha\left(y_{3}\right)<a .
$$

Choose

Define the norm

$$
X=\left\{\{x(n)\}: \begin{array}{c}
x(n) \in R, n \in N_{0} \\
\text { there exist the limits } \\
\lim _{n \rightarrow \infty} \frac{x(n)}{\sum_{s=0}^{n-1} \frac{1}{\phi^{-1}(p(s))}+1}, \\
\lim _{n \rightarrow \infty} \phi^{-1}(p(n)) \Delta x(n)
\end{array}\right\} .
$$

$$
\|x\|=\max \left\{\sup _{n \in N_{0}} \frac{|x(n)|}{1+\sum_{s=0}^{n-1} \frac{1}{\phi^{-1}(p(s))}}, \sup _{n \in N_{0}} \phi^{-1}(p(n))|\Delta x(n)|\right\} .
$$

It is easy to see that $X$ is a real Banach space.

Denote $[a, b]=\{a, a+1, \cdots, b\}$ for $a \leq b$ and $[a, b]=\varnothing$ for $a>b$ with $a, b \in N_{0}$, $N$ denotes the set of all positive integeres. Let $k_{1}, k_{2} \in N$ with $k_{1}<k_{2}$. Let

$$
\mu=\frac{1}{\max \left\{1, \phi^{-1}\left(p\left(k_{1}-1\right)\right)\right\}\left(1+\sum_{n=0}^{k_{2}-1} \frac{1}{\phi^{-1}(p(n))}\right)} .
$$

Choose

$$
P=\left\{x \in X: \begin{array}{l}
x(n) \geq 0 \text { for all } n \in N_{0}, \\
\min _{n \in\left[k_{1}, k_{2}\right]} \frac{x(n)}{1+\sum_{s=0}^{n-1} \frac{1}{\phi^{-1}(p(s))}} \geq \mu \sup _{n \in N_{0}} \frac{x(n)}{1+\sum_{s=0}^{n-1} \frac{1}{\phi^{-1}(p(s))}} .
\end{array}\right\},
$$

It is easy to see that $P$ is a nontrivial cone in $X$. 
Let $h(n) \not \equiv 0\left(n \in N_{0}\right)$ be a nonnegative sequence with $\sum_{n \in N_{0}} h(n)$ converging, consider the following BVP

$$
\left\{\begin{array}{l}
\Delta[p(n) \phi(\Delta y(n))]+h(n)=0, n \in N_{0} \\
y(0)-\sum_{n=1}^{\infty} \alpha_{n} y(n)=0 \\
\lim _{n \rightarrow \infty} \frac{y(n)}{1+\sum_{s=0}^{n-1} \frac{1}{\phi^{-1}(p(s))}}-\sum_{n=1}^{\infty} \beta_{n} y(n)=0
\end{array}\right.
$$

Set

$$
\delta=\frac{\phi\left(\frac{\sum_{n=1}^{\infty} \beta_{n}}{1-\sum_{n=1}^{\infty} \alpha_{n}} \sum_{n=1}^{\infty} \alpha_{n} \sum_{j=0}^{n-1} \phi^{-1}\left(\frac{1}{p(j)}\right)+\sum_{n=1}^{\infty} \beta_{n} \sum_{j=0}^{n-1} \phi^{-1}\left(\frac{1}{p(j)}\right)\right)}{1-\phi\left(\frac{\sum_{n=1}^{\infty} \beta_{n}}{1-\sum_{n=1}^{\infty} \alpha_{n}} \sum_{n=1}^{\infty} \alpha_{n} \sum_{j=0}^{n-1} \phi^{-1}\left(\frac{1}{p(j)}\right)+\sum_{n=1}^{\infty} \beta_{n} \sum_{j=0}^{n-1} \phi^{-1}\left(\frac{1}{p(j)}\right)\right)} .
$$

Lemma 2. If $y$ is a solution of $B V P(6)$, then $y(n) \geq 0$ and $\Delta y(n) \geq 0$ for all $n \in N_{0}$ and there exists an unique number $A_{h} \in\left[0, \delta \sum_{s=0}^{\infty} h(s)\right]$ such that

$$
\begin{gathered}
y(n)=\frac{1}{1-\sum_{n=1}^{\infty} \alpha_{n}} \\
\sum_{n=1}^{\infty} \alpha_{n} \sum_{j=0}^{n-1} \phi^{-1}\left(\frac{A_{h}+\sum_{s=j}^{\infty} h(s)}{p(j)}\right)+\sum_{j=0}^{n-1} \phi^{-1}\left(\frac{A_{h}+\sum_{s=j}^{\infty} h(s)}{p(j)}\right) .
\end{gathered}
$$

Proof. Since $\Delta[p(n) \phi(\Delta y(n))]=-h(n) \leq 0$ for all $n \in N_{0}$ and $\sum_{n \in N_{0}} h(n)$ converges, we get that $p(n) \phi(\Delta y(n))$ is decreasing and there exists the limit $\lim _{n \rightarrow \infty} p(n) \phi(\Delta y(n))$. Then $\phi^{-1}(p(n)) \Delta y(n)$ is decreasing and there exists the limit $\lim _{n \rightarrow \infty} \phi^{-1}(p(n)) \Delta y(n)$.

Since there exists the $\operatorname{limit}_{n \rightarrow \infty} \phi^{-1}(p(n)) \Delta y(n)=c$, we can prove

$$
\lim _{n \rightarrow \infty} \frac{y(n)}{1+\sum_{s=0}^{n-1} \frac{1}{\phi^{-1}(p(s))}}=c .
$$

In fact, if $c=0$, then for any $\epsilon>0$ there exists $H>0$ such that

$$
\phi^{-1}(p(n))|\Delta y(n)|<\frac{\epsilon}{2}, n \geq H .
$$

It follows that

$$
|y(n)| \leq|y(H)|+\sum_{s=H}^{n-1}|\Delta y(s)| \leq|y(H)|+\frac{\epsilon}{2} \sum_{s=H}^{n-1} \frac{1}{\phi^{-1}(p(s))}, n \geq H .
$$

Then

$$
\frac{|y(n)|}{1+\sum_{s=0}^{n-1} \frac{1}{\phi^{-1}(p(s))}}
$$




$$
\begin{gathered}
\leq \frac{|y(H)|}{1+\sum_{s=0}^{n-1} \frac{1}{\phi^{-1}(p(s))}}+\frac{1}{1+\sum_{s=0}^{n-1} \frac{1}{\phi^{-1}(p(s))}} \frac{\epsilon}{2} \sum_{s=H}^{n-1} \frac{1}{\phi^{-1}(p(s))} \\
<\frac{|y(H)|}{1+\sum_{s=0}^{n-1} \frac{1}{\phi^{-1}(p(s))}}+\frac{\epsilon}{2}, \quad n \geq H .
\end{gathered}
$$

Since $\sum_{s=0}^{\infty} \frac{1}{\phi^{-1}(p(s))}=\infty$, we can choose $H^{\prime}>H$ large enough so that

$$
\frac{|y(n)|}{1+\sum_{s=0}^{n-1} \frac{1}{\phi^{-1}(p(s))}} \leq \frac{|y(H)|}{1+\sum_{s=0}^{n-1} \frac{1}{\phi^{-1}(p(s))}}+\frac{\epsilon}{2}<\epsilon, n \geq H^{\prime},
$$

which implies that

$$
\lim _{n \rightarrow \infty} \frac{y(n)}{1+\sum_{s=0}^{n-1} \frac{1}{\phi^{-1}(p(s))}}=0 .
$$

If $c \neq 0$, then $\lim _{t \rightarrow \infty}\left(\phi^{-1}(p(n)) \Delta y(n)-c\right)=0$. It follows that

$$
\lim _{t \rightarrow \infty} \phi^{-1}(p(n)) \Delta\left[y(n)-c \sum_{s=0}^{n-1} \frac{1}{\phi^{-1}(p(s))}\right]=0 .
$$

Then we get similarly that

$$
\lim _{t \rightarrow \infty} \frac{y(n)-c \sum_{s=0}^{n-1} \frac{1}{\phi^{-1}(p(s))}}{1+\sum_{s=0}^{n-1} \frac{1}{\phi^{-1}(p(s))}}=0 .
$$

Together with $\sum_{s=0}^{\infty} \frac{1}{\phi^{-1}(p(s))}=\infty$, it follows that

$$
\lim _{n \rightarrow \infty} \frac{y(n)}{1+\sum_{s=0}^{n-1} \frac{1}{\phi^{-1}(p(s))}}=c .
$$

It follows from 2.2 that there exist $A, B \in R$ such that

$$
p(n) \phi(\Delta y(n))=A+\sum_{s=n}^{\infty} h(s),
$$

and

$$
y(n)=B+\sum_{j=0}^{n-1} \phi^{-1}\left(\frac{1}{p(j)}\left(A+\sum_{s=n}^{\infty} h(s)\right)\right), n \in N_{0} .
$$

By the boundary conditions in 2.2, we get

$$
B=B \sum_{n=1}^{\infty} \alpha_{n}+\sum_{n=1}^{\infty} \alpha_{n} \sum_{j=0}^{n-1} \phi^{-1}\left(\frac{1}{p(j)}\left(A+\sum_{s=j}^{\infty} h(s)\right)\right) .
$$


It follows that

$$
B=\frac{1}{1-\sum_{n=1}^{\infty} \alpha_{n}} \sum_{n=1}^{\infty} \alpha_{n} \sum_{j=0}^{n-1} \phi^{-1}\left(\frac{1}{p(j)}\left(A+\sum_{s=j}^{\infty} h(s)\right)\right) .
$$

So

$$
\begin{gathered}
y(n)=\frac{1}{1-\sum_{n=1}^{\infty} \alpha_{n}} \sum_{n=1}^{\infty} \alpha_{n} \sum_{j=0}^{n-1} \phi^{-1}\left(\frac{1}{p(j)}\left(A+\sum_{s=j}^{\infty} h(s)\right)\right) \\
+\sum_{j=0}^{n-1} \phi^{-1}\left(\frac{1}{p(j)}\left(A+\sum_{s=j}^{\infty} h(s)\right) .\right.
\end{gathered}
$$

From 2.5, one sees that $\lim _{n \rightarrow \infty} p(n) \phi(\Delta y(n))=A$.

Then $\lim _{n \rightarrow \infty} \phi^{-1}(p(n)) \Delta y(n)=\phi^{-1}(A)$. So

$$
\lim _{n \rightarrow \infty} \frac{y(n)}{1+\sum_{s=0}^{n-1} \frac{1}{\phi^{-1}(p(s))}}=\phi^{-1}(A) .
$$

Boundary conditions in 2.2 imply that

$$
\begin{aligned}
\phi^{-1}(A)= & \frac{\sum_{n=1}^{\infty} \beta_{n}}{1-\sum_{n=1}^{\infty} \alpha_{n}} \sum_{n=1}^{\infty} \alpha_{n} \sum_{j=0}^{n-1} \phi^{-1}\left(\frac{A+\sum_{s=j}^{\infty} h(s)}{p(j)}\right) \\
& +\sum_{n=1}^{\infty} \beta_{n} \sum_{j=0}^{n-1} \phi^{-1}\left(\frac{A+\sum_{s=j}^{\infty} h(s)}{p(j)}\right) .
\end{aligned}
$$

Set

$$
\begin{aligned}
G(x)= & 1-\frac{\sum_{n=1}^{\infty} \beta_{n}}{1-\sum_{n=1}^{\infty} \alpha_{n}} \sum_{n=1}^{\infty} \alpha_{n} \sum_{j=0}^{n-1} \phi^{-1}\left(\frac{1}{p(j)}\left(1+\frac{\sum_{s=j}^{\infty} h(s)}{x}\right)\right) \\
& -\sum_{n=1}^{\infty} \beta_{n} \sum_{j=0}^{n-1} \phi^{-1}\left(\frac{1}{p(j)}\left(1+\frac{\sum_{s=j}^{\infty} h(s)}{x}\right)\right) .
\end{aligned}
$$

It is easy to see that $G(x)$ is continuous on $(-\infty, 0)$ and $(0, \infty)$, respectively, $G(x)$ is increasing on $(-\infty, 0)$ and $(0, \infty)$, respectively.

Since

$$
=1-\frac{\sum_{n=1}^{\infty} \beta_{n}}{1-\sum_{n=1}^{\infty} \alpha_{n}} \sum_{n=1}^{\infty} \alpha_{n} \sum_{j=0}^{n-1} \phi^{-1}\left(\frac{1}{p(j)}\right)-\sum_{n=1}^{\infty} \beta_{n} \sum_{j=0}^{n-1} \phi^{-1}\left(\frac{1}{p(j)}\right)>0,
$$


and

$$
\lim _{x \rightarrow 0^{-}} G(x)=+\infty
$$

we get $G(x)$ has no zero point on $(-\infty, 0)$. On the other hand, we have

$$
\begin{gathered}
G\left(\delta \sum_{s=0}^{\infty} h(s)\right) \\
=1-\frac{\sum_{n=1}^{\infty} \beta_{n}}{1-\sum_{n=1}^{\infty} \alpha_{n}} \sum_{n=1}^{\infty} \alpha_{n} \sum_{j=0}^{n-1} \phi^{-1}\left(\frac{1}{p(j)}\left(1+\frac{\sum_{s=j}^{\infty} h(s)}{\delta \sum_{s=0}^{\infty} h(s)}\right)\right) \\
-\sum_{n=1}^{\infty} \beta_{n} \sum_{j=0}^{n-1} \phi^{-1}\left(\frac{1}{p(j)}\left(1+\frac{\sum_{s=j}^{\infty} h(s)}{\delta \sum_{s=0}^{\infty} h(s)}\right)\right) \\
\geq 1-\frac{\sum_{n=1}^{\infty} \beta_{n}}{1-\sum_{n=1}^{\infty} \alpha_{n}} \sum_{n=1}^{\infty} \alpha_{n} \sum_{j=0}^{n-1} \phi^{-1}\left(\frac{1}{p(j)}\left(1+\frac{1}{\delta}\right)\right) \\
-\sum_{n=1}^{\infty} \beta_{n} \sum_{j=0}^{n-1} \phi^{-1}\left(\frac{1}{p(j)}\left(1+\frac{1}{\delta}\right)\right) \\
=1-\phi^{-1}\left(1+\frac{1}{\delta}\right)\left(\frac{\sum_{n=1}^{\infty} \beta_{n}}{1-\sum_{n=1}^{\infty} \alpha_{n}} \sum_{n=1}^{\infty} \alpha_{n} \sum_{j=0}^{n-1} \phi^{-1}\left(\frac{1}{p(j)}\right)\right. \\
\left.+\sum_{n=1}^{\infty} \beta_{n} \sum_{j=0}^{n-1} \phi^{-1}\left(\frac{1}{p(j)}\right)\right)
\end{gathered}
$$

and

$$
\lim _{x \rightarrow 0^{+}} G(x)=-\infty
$$

Hence we find that there exists an unique $\xi \in\left(0, \delta \sum_{s=0}^{\infty} h(s)\right]$ such that $G(\xi)=0$. It follows that there exists unique $A_{h} \in\left[0, \delta \sum_{s=0}^{\infty} h(s)\right]$ satisfying 2 . Hence 2.4 holds for $A_{h} \in\left[0, \delta \sum_{s=0}^{\infty} h(s)\right]$. It is easy to see from 2.4 that $y(n) \geq 0$ and $\Delta y(n) \geq 0$ for all $n \in N_{0}$. The proof is complete.

Lemma 3. Choose integers $k_{1}, k_{2} \in N_{0}$ with $1<k_{1}+1<k_{2}$. Let $\mu$ be defined by 2.1. Suppose $y$ is a solution of $B V P(6)$. Then

$$
\min _{\left.n \in\left[k_{1}, k_{2}\right]\right)} \frac{y(n)}{1+\sum_{s=0}^{n-1} \frac{1}{\phi^{-1}(p(s))}} \geq \mu \sup _{n \in N_{0}} \frac{y(n)}{1+\sum_{s=0}^{n-1} \frac{1}{\phi^{-1}(p(s))}} .
$$


Proof. Since $\Delta[p(n) \phi(\Delta y(n))]=-h(n) \leq 0$ for all $n \in N_{0}$, we see that $p(n) \phi(\Delta y(n))$ is decreasing. Then $\phi^{-1}(p(n)) \Delta y(n)$ is decreasing.

It follows from Lemma 1 that $y(n) \geq 0$ and $\Delta y(n) \geq 0$ for all $n \in N_{0}$ and there exists the limit

$$
\sup _{n \in N_{0}} \frac{y(n)}{1+\sum_{s=0}^{n-1} \frac{1}{\phi^{-1}(p(s))}} .
$$

To complete the proof of 2.7, we consider two cases:

Case 1. there is $n_{0} \in N_{0}$ such that

$$
\sup _{n \in N_{0}} \frac{y(n)}{1+\sum_{s=0}^{n-1} \frac{1}{\phi^{-1}(p(s))}}=\frac{y\left(n_{0}\right)}{1+\sum_{s=0}^{n_{0}-1} \frac{1}{\phi^{-1}(p(s))}} .
$$

For $n_{1}, n, n_{2} \in N_{0}$ with $n_{1}<n<n_{2}$, we have

$$
\begin{gathered}
\left(\sum_{s=0}^{n-1} \frac{1}{\phi^{-1}(p(s))}-\sum_{s=0}^{n_{1}-1} \frac{1}{\phi^{-1}(p(s))}\right) \\
\frac{y\left(n_{2}\right)-y(n)}{\sum_{s=0}^{n_{2}-1} \frac{1}{\phi^{-1}(p(s))}-\sum_{s=0}^{n-1} \frac{1}{\phi^{-1}(p(s))}+y\left(n_{1}\right)-y(n)} \\
=\frac{\left(\sum_{s=0}^{n-1} \frac{1}{\phi^{-1}(p(s))}-\sum_{s=0}^{n_{1}-1} \frac{1}{\phi^{-1}(p(s))}\right)\left(y\left(n_{2}\right)-y(n)\right)}{\sum_{s=0}^{n_{2}-1} \frac{1}{\phi^{-1}(p(s))}-\sum_{s=0}^{n-1} \frac{1}{\phi^{-1}(p(s))}} \\
+\frac{\left(\sum_{s=0}^{n_{2}-1} \frac{1}{\phi^{-1}(p(s))}-\sum_{s=0}^{n-1} \frac{1}{\phi^{-1}(p(s))}\right)\left(y\left(n_{1}\right)-y(n)\right)}{\sum_{s=0}^{n_{2}-1} \frac{1}{\phi^{-1}(p(s))}-\sum_{s=0}^{n-1} \frac{1}{\phi^{-1}(p(s))}} \\
=\frac{\left.1 \sum_{s=0}^{n-1} \frac{1}{\phi^{-1}(p(s))}-\sum_{s=0}^{n_{1}-1} \frac{1}{\phi^{-1}(p(s))}\right) \sum_{s=n}^{n_{2}-1} \frac{1}{\phi^{-1}(p(s))} \phi^{-1}(p(s)) \Delta y(s)}{\sum_{s=0}^{n_{2}-1} \frac{1}{\phi^{-1}(p(s))}-\sum_{s=0}^{n-1} \frac{1}{\phi^{-1}(p(s))}} \\
-\frac{\left(\sum_{s=0}^{n_{2}-1} \frac{1}{\phi^{-1}(p(s))}-\sum_{s=0}^{n-1} \frac{1}{\phi^{-1}(p(s))}\right) \sum_{s=n_{1}}^{n-1} \frac{1}{\phi^{-1}(p(s))} \phi^{-1}(p(s)) \Delta y(s)}{\sum_{s=0}^{n_{2}-1} \frac{1}{\phi^{-1}(p(s))}-\sum_{s=0}^{n-1} \frac{1}{\phi^{-1}(p(s))}} .
\end{gathered}
$$

Since $\phi^{-1}(p(n)) \Delta y(n)$ is decreasing, we get $\phi^{-1}(p(s)) \Delta y(s) \leq \phi^{-1}(p(k)) \Delta y(k)$ for all $s \geq k$. So there there is $\lambda$ such that

$$
\phi^{-1}(p(s)) \Delta y(s) \leq \phi^{-1}(p(k)) \Delta y(k), s \geq k .
$$

Then we get

$$
\frac{\left(\sum_{s=0}^{n-1} \frac{1}{\phi^{-1}(p(s))}-\sum_{s=0}^{n_{1}-1} \frac{1}{\phi^{-1}(p(s))}\right) \sum_{s=n}^{n_{2}-1} \frac{1}{\phi^{-1}(p(s))} \phi^{-1}(p(s)) \Delta y(s)}{\sum_{s=0}^{n_{2}-1} \frac{1}{\phi^{-1}(p(s))}-\sum_{s=0}^{n-1} \frac{1}{\phi^{-1}(p(s))}}
$$




$$
\begin{aligned}
& =\frac{\sum_{s=n_{1}}^{n-1} \frac{1}{\phi^{-1}(p(s))} \sum_{s=n}^{n_{2}-1} \frac{1}{\phi^{-1}(p(s))} \phi^{-1}(p(s)) \Delta y(s)}{\sum_{s=n}^{n_{2}-1} \frac{1}{\phi^{-1}(p(s))}} \\
& \leq \frac{\lambda \sum_{s=n_{1}}^{n-1} \frac{1}{\phi^{-1}(p(s))} \sum_{s=n}^{n_{2}-1} \frac{1}{\phi^{-1}(p(s))}}{\sum_{s=n}^{n_{2}-1} \frac{1}{\phi^{-1}(p(s))}} \\
& =\frac{\lambda \sum_{s=n}^{n_{2}-1} \frac{1}{\phi^{-1}(p(s))} \sum_{s=n_{1}}^{n-1} \frac{1}{\phi^{-1}(p(s))}}{\sum_{s=n}^{n_{2}-1} \frac{1}{\phi^{-1}(p(s))}} \\
& \leq \frac{\sum_{s=n}^{n_{2}-1} \frac{1}{\phi^{-1}(p(s))} \sum_{s=n_{1}}^{n-1} \frac{1}{\phi^{-1}(p(s))} \phi^{-1}(p(s)) \Delta y(s)}{\sum_{s=n}^{n_{2}-1} \frac{1}{\phi^{-1}(p(s))}}
\end{aligned}
$$

So

It follows that

$$
\left(\sum_{s=n_{1}}^{n-1} \frac{1}{\phi^{-1}(p(s))}\right) \frac{y\left(n_{2}\right)-y(n)}{\sum_{s=n}^{n_{2}-1} \frac{1}{\phi^{-1}(p(s))}}+y\left(n_{1}\right)-y(n) \leq 0 .
$$

$$
y(n) \geq \frac{\sum_{s=n}^{n_{2}-1} \frac{1}{\phi^{-1}(p(s))}}{\sum_{s=n_{1}}^{n_{2}-1} \frac{1}{\phi^{-1}(p(s))}} y\left(n_{1}\right)+\frac{\sum_{s=n_{1}}^{n-1} \frac{1}{\phi^{-1}(p(s))}}{\sum_{s=n_{1}}^{n_{2}-1} \frac{1}{\phi^{-1}(p(s))}} y\left(n_{2}\right) .
$$

If $n_{0}=k_{1}$, we get

$$
\begin{gathered}
\min _{n \in\left[k_{1}, k_{2}\right]} \frac{y(n)}{1+\sum_{s=0}^{n-1} \frac{1}{\phi^{-1}(p(s))}} \geq \frac{y\left(k_{1}\right)}{1+\sum_{s=0}^{k_{2}-1} \frac{1}{\phi^{-1}(p(s))}} \\
=\frac{y\left(n_{0}\right)}{1+\sum_{s=0}^{n_{0}-1} \frac{1}{\phi^{-1}(p(s))}} \frac{1+\sum_{s=0}^{k_{1}-1} \frac{1}{\phi^{-1}(p(s))}}{1+\sum_{s=0}^{k_{2}-1} \frac{1}{\phi^{-1}(p(s))}} \\
\geq \frac{1}{\phi^{-1}\left(p\left(k_{1}-1\right)\right)\left(1+\sum_{s=0}^{k_{2}-1} \frac{1}{\phi^{-1}(p(s))}\right)} \sup _{n \in N_{0}} \frac{y(n)}{1+\sum_{s=0}^{n-1} \frac{1}{\phi^{-1}(p(s))}} \\
\geq \mu \sup _{n \in N_{0}} \frac{y(n)}{1+\sum_{s=0}^{n-1} \frac{1}{\phi^{-1}(p(s))}}
\end{gathered}
$$

If $n_{0}>k_{1}$, choose $n_{1}=k_{1}-1, n=k_{1}$ and $n_{2}=n_{0}$, by using 2.8 we have

$$
\begin{aligned}
y\left(k_{1}\right) & =\frac{\sum_{s=k_{1}}^{n_{0}-1} \frac{1}{\phi^{-1}(p(s))}}{\sum_{s=k_{1}-1}^{n_{0}-1} \frac{1}{\phi^{-1}(p(s))}} y\left(k_{1}-1\right)+\frac{\sum_{s=k_{1}-1}^{k_{1}-1} \frac{1}{\phi^{-1}(p(s))}}{\sum_{s=k_{1}-1}^{n_{0}-1} \frac{1}{\phi^{-1}(p(s))}} y\left(n_{0}\right) \\
& \geq \frac{\sum_{s=k_{1}-1 \frac{1}{k_{1}-1}(p(s))}^{k^{-1}}}{\sum_{s=k_{1}-1}^{n_{0}-1} \frac{1}{\phi^{-1}(p(s))}} y\left(n_{0}\right) .
\end{aligned}
$$


Then

$$
\begin{aligned}
& \min _{n \in\left[k_{1}, k_{2}\right]} \frac{y(n)}{1+\sum_{s=0}^{n-1} \frac{1}{\phi^{-1}(p(s))}} \\
& \geq \frac{y\left(k_{1}\right)}{1+\sum_{s=0}^{k_{2}-1} \frac{1}{\phi^{-1}(p(s))}} \\
& \geq \frac{1+\sum_{s=0}^{n_{0}-1} \frac{1}{\phi^{-1}(p(s))} \sum_{s=k_{1}-1 \frac{1}{\phi^{-1}(p(s))}}^{k_{1}-1} \frac{y\left(n_{0}\right)}{1+\sum_{s=0}^{n_{0}-1} \frac{1}{\phi^{-1}(p(s))}}}{1+\sum_{s=0}^{k_{2}-1} \frac{1}{\phi^{-1}(p(s))} \sum_{s=k_{1}-1}^{n_{\phi^{-1}(p(s))}}} \\
& \geq \frac{\sup _{n \in N_{0}} \frac{y(n)}{1+\sum_{s=0}^{n-1} \frac{1}{\phi^{-1}(p(s))}}}{\phi^{-1}\left(p\left(k_{1}-1\right)\right)\left(1+\sum_{s=0}^{k_{2}-1} \frac{1}{\phi^{-1}(p(s))}\right.} \\
& \geq \mu \sup _{n \in N_{0}} \frac{y(n)}{1+\sum_{s=0}^{n-1} \frac{1}{\phi^{-1}(p(s))}}
\end{aligned}
$$

If $n_{0}<k_{1}$, we have

$$
\begin{aligned}
& \min _{n \in\left[k_{1}, k_{2}\right]} \frac{y(n)}{1+\sum_{s=0}^{n-1} \frac{1}{\phi^{-1}(p(s))}} \\
& \geq \frac{y\left(k_{1}\right)}{1+\sum_{s=0}^{k_{2}-1} \frac{1}{\phi^{-1}(p(s))}} \\
& \geq \frac{1}{1+\sum_{s=0}^{k_{2}-1} \frac{1}{\phi^{-1}(p(s))}} y\left(n_{0}\right) \\
& \geq \frac{1+\sum_{s=0}^{n_{0}-1} \frac{1}{\phi^{-1}(p(s))}}{1+\sum_{s=0}^{k_{2}-1} \frac{1}{\phi^{-1}(p(s))}} \frac{y\left(n_{0}\right)}{1+\sum_{s=0}^{n_{0}-1} \frac{1}{\phi^{-1}(p(s))}} \\
& \geq \frac{1}{1+\sum_{s=0}^{k_{2}-1} \frac{1}{\phi^{-1}(p(s))}} \sup _{n \in N_{0}} \frac{y(n)}{1+\sum_{s=0}^{n-1} \frac{1}{\phi^{-1}(p(s))}} \\
& \geq \mu \sup _{n \in N_{0}} \frac{y(n)}{1+\sum_{s=0}^{n-1} \frac{1}{\phi^{-1}(p(s))}} .
\end{aligned}
$$

Case 2. $\sup _{n \in N_{0}} \frac{y(n)}{1+\sum_{s=0}^{n-1} \frac{1}{\phi^{-1}(p(s))}}=\lim _{n \rightarrow \infty} \frac{y(n)}{1+\sum_{s=0}^{n-1} \frac{1}{\phi^{-1}(p(s))}}$.

Choose $n^{\prime}>k_{2}$, similarly to Case 1 we can prove that

$$
\min _{n \in\left[k_{1}, k_{2}\right]} \frac{y(n))}{1+\sum_{s=0}^{n-1} \frac{1}{\phi^{-1}(p(s))}} \geq \mu \frac{y\left(n^{\prime}\right)}{1+n^{\prime}} .
$$


Let $n^{\prime} \rightarrow \infty$, one sees

$$
\min _{n \in\left[k_{1}, k_{2}\right]} \frac{y(n))}{1+\sum_{s=0}^{n-1} \frac{1}{\phi^{-1}(p(s))}} \geq \mu \sup _{n \in N_{0}} \frac{y(n)}{1+\sum_{s=0}^{n-1} \frac{1}{\phi^{-1}(p(s))}} .
$$

From Cases 1 and 2, we get 2.7. The proof is complete.

Define the functionals on $P: P \rightarrow R$ by

$$
\begin{aligned}
& \gamma(x)=\sup _{n \in N_{0}} \phi^{-1}(p(n))|\Delta x(n)|, x \in P, \\
& \beta(x)=\sup _{n \in N_{0}} \frac{x(n)}{1+\sum_{s=0}^{n-1} \frac{1}{\phi^{-1}(p(s))}}, x \in P, \\
& \theta(x)=\sup _{n \in N_{0}} \frac{x(n)}{1+\sum_{s=0}^{n-1} \frac{1}{\phi^{-1}(p(s))}}, x \in P, \\
& \alpha(x)=\min _{n \in\left[k_{1}, k_{2}\right]} \frac{x(n)}{1+\sum_{s=0}^{n-1} \frac{1}{\phi^{-1}(p(s))}}, x \in P, \\
& \psi(x)=\min _{n \in\left[k_{1}, k_{2}\right]} \frac{x(n)}{1+\sum_{s=0}^{n-1} \frac{1}{\phi^{-1}(p(s))}}, x \in P .
\end{aligned}
$$

Lemma 4. If $y$ is a solution of $B V P(6)$, we have $\|y\| \leq M \gamma(y)$ for all $y \in P$, where

$$
M=\max \left\{1, \sup _{n \in N_{0}} \frac{\sum_{i=0}^{n-1} \frac{1}{\phi^{-1}(p(i))}+\frac{\sum_{n \in N} \alpha_{n} \sum_{i=0}^{n-1} \frac{1}{\phi^{-1}(p(i))}}{1-\sum_{i=1}^{\infty} \alpha_{i}}}{1+\sum_{i=0}^{n-1} \frac{1}{\phi^{-1}(p(i))}}\right\} .
$$

Proof. Since $y$ is the solution of $\operatorname{BVP}(6)$, we get

$$
\begin{gathered}
|y(n)|=|y(n)-y(0)+y(0)| \\
\leq\left|\sum_{i=0}^{n-1} \Delta y(i)\right|+\left|\frac{y(0)-\sum_{n \in N} \alpha_{n} y(0)}{1-\sum_{n \in N} \alpha_{n}}\right| \\
=\left|\sum_{i=0}^{n-1} \frac{1}{\phi^{-1}(p(i))} \phi^{-1}(p(i)) \Delta y(i)\right|+\frac{\sum_{n \in N} \alpha_{n}|y(n)-y(0)|}{1-\sum_{n \in N} \alpha_{n}} \\
\leq \sum_{i=0}^{n-1} \frac{1}{\phi^{-1}(p(i))} \sup _{n \in N_{0}} \phi^{-1}(p(n))|\Delta y(n)|+\left|\frac{\sum_{n \in N} \alpha_{n}[y(n)-y(0)]}{1-\sum_{i=1}^{\infty} \alpha_{i}}\right| \\
\leq\left(\sum_{i=0}^{n-1} \frac{1}{\phi^{-1}(p(i))}+\frac{\sum_{n \in N} \alpha_{n} \sum_{i=0}^{n-1} \frac{1}{\phi^{-1}(p(i))}}{1-\sum_{i=1}^{\infty} \alpha_{i}}\right) \sup _{n \in N_{0}} \phi^{-1}(p(n))|\Delta y(n)| .
\end{gathered}
$$


It follows that

$$
\begin{gathered}
\frac{\frac{y(n)}{1+\sum_{i=0}^{n-1} \frac{1}{\phi^{-1}(p(i))}}}{1+\sum_{i=0}^{n-1} \frac{1}{\phi^{-1}(p(i))}} \\
\leq \frac{\sum_{i=0}^{n-1} \frac{1}{\phi^{-1}(p(i))}+\frac{\sum_{n \in N} \alpha_{n} \sum_{i=0}^{n-1} \frac{1}{\phi^{-1}(p(i))}}{1-\sum_{i=1}^{\infty} \alpha_{i}}}{n \in N_{0}} \phi^{-1}(p(n))|\Delta y(n)| \\
\leq \sup _{n \in N_{0}} \frac{\sum_{i=0}^{n-1} \frac{1}{\phi^{-1}(p(i))}+\frac{\sum_{n \in N} \alpha_{n} \sum_{i=0}^{n-1} \frac{1}{\phi^{-1}(p(i))}}{1-\sum_{i=1}^{\infty} \alpha_{i}}}{1+\sum_{i=0}^{n-1} \frac{1}{\phi^{-1}(p(i))}} \sup _{n \in N_{0}} \phi^{-1}(p(n))|\Delta y(n)| .
\end{gathered}
$$

we get that

$$
\begin{gathered}
\|y\|=\max \left\{\sup _{n \in N_{0}} \frac{|y(n)|}{1+\sum_{i=0}^{n-1} \frac{1}{\phi^{-1}(p(i))}}, \sup _{n \in N_{0}} \phi^{-1}(p(n))|\Delta y(n)|\right\} \\
\leq \max \left\{1, \sup _{n \in N_{0}} \frac{\sum_{i=0}^{n-1} \frac{1}{\phi^{-1}(p(i))}+\frac{\sum_{n \in N} \alpha_{n} \sum_{i=0}^{n-1} \frac{1}{\phi^{-1}(p(i))}}{1-\sum_{i=1}^{\infty} \alpha_{i}}}{1+\sum_{i=0}^{n-1} \frac{1}{\phi^{-1}(p(i))}}\right\} \\
\sup _{n \in N_{0}} \phi^{-1}(p(n))|\Delta y(n)| \\
=M \gamma(y) .
\end{gathered}
$$

Then $\|y\| \leq M \gamma(y)$ for all solutions $y$ of $\mathrm{BVP}(6)$. The proof is complete.

For $x \in P$, define $(T x)(n)$ by

$$
\begin{aligned}
(T x)(n)= & \frac{1}{1-\sum_{n=1}^{\infty} \alpha_{n}} \sum_{n=1}^{\infty} \alpha_{n} \sum_{j=0}^{n-1} \phi^{-1}\left(\frac{A_{x}+\sum_{s=j}^{\infty} f(s, x(s), \Delta x(s))}{p(j)}\right) \\
& +\sum_{j=0}^{n-1} \phi^{-1}\left(\frac{A_{x}+\sum_{s=j}^{\infty} f(s, x(s), \Delta x(s))}{p(j)}\right), n \in N_{0},
\end{aligned}
$$

where $A_{x}$ satisfies

$$
\begin{aligned}
\phi^{-1}\left(A_{x}\right)= & \frac{\sum_{n=1}^{\infty} \beta_{n}}{1-\sum_{n=1}^{\infty} \alpha_{n}} \sum_{n=1}^{\infty} \alpha_{n} \sum_{j=0}^{n-1} \phi^{-1}\left(\frac{A_{x}+\sum_{s=j}^{\infty} f(s, x(s), \Delta x(s))}{p(j)}\right) \\
& +\sum_{n=1}^{\infty} \beta_{n} \sum_{j=0}^{n-1} \phi^{-1}\left(\frac{A_{x}+\sum_{s=j}^{\infty} f(s, x(s), \Delta x(s))}{p(j)}\right) .
\end{aligned}
$$


One sees easily that

$$
\left\{\begin{array}{l}
\Delta[\phi(\Delta(T x)(n))]+f(n, x(n), \Delta x(n))=0, n \in N_{0}, \\
(T x)(0)-\sum_{i=1}^{\infty} \alpha_{i}(T x)(i)=0, \\
\lim _{n \rightarrow \infty} \frac{(T x)(n)}{1+\sum_{i=0}^{n-1} \frac{1}{\phi^{-1}(p(i))}}-\sum_{i=1}^{\infty} \beta_{i}(T x)(i)=0 .
\end{array}\right.
$$

Let $\delta$ be defined by 2.3. By Lemma 2, we have

$$
A_{x} \in\left[0, \delta \sum_{n=0}^{\infty} f(n, x(n), \Delta x(n))\right] \text {. }
$$

Lemma 5. Let $V=\{x \in X:\|x\|<l\}(l>0)$. If $\left\{\frac{x(n)}{1+\sum_{s=0}^{n-1} \frac{1}{\phi^{-1}(p(s))}}: x \in V\right\}$ and $\left\{\phi^{-1}(p(n)) \Delta x(n): x \in V\right\}$ are both equiconvergent at infinity, where

$$
V_{1}=:\left\{\frac{x(n)}{1+\sum_{s=0}^{n-1} \frac{1}{\phi^{-1}(p(s))}}: x \in V\right\} \bigcup\left\{\phi^{-1}(p(n)) \Delta x(n): x \in V\right\}
$$

is called equiconvergent at infinity if and only iffor all $\epsilon>0$, there exists $N=N(\epsilon)>$ 0 such that for all $x \in V$, it holds that

$$
\left|\frac{x\left(n_{1}\right)}{1+\sum_{s=0}^{n_{1}-1} \frac{1}{\phi^{-1}(p(s))}}-\frac{x\left(n_{2}\right)}{1+\sum_{s=0}^{n_{2}-1} \frac{1}{\phi^{-1}(p(s))}}\right|<\epsilon,
$$

and

$$
\left|\phi^{-1}\left(p\left(n_{1}\right)\right) \Delta x\left(n_{1}\right)-\phi^{-1}\left(p\left(n_{2}\right)\right) \Delta x\left(n_{2}\right)\right|<\epsilon n_{1}, n_{2}>N .
$$

Then $V$ is pre-compact on $X$.

Proof. The proof is similar to that of the proof of a Lemma in [14] and is omitted.

Lemma 6. The following facts hold.

(i) $T x \in P$ for each $x \in P$;

(ii) $x$ is a solution of $B V P(3)$ if and only if $x$ is a solution of the operator equation $x=T x$;

(iii) $T: P \rightarrow P$ is completely continuous;

Proof. (i) Note the definition of $P$. For $x \in P$, Lemma 2 and Lemma 3 imply that $T x \in P$.

(ii) It is easy to see that $x$ is a solution of $\mathrm{BVP}(3)$ if and only if $x$ is a solution of the operator equation $x=T x$.

(iii) It suffices to prove that $T$ is continuous on $P$ and $T$ maps bounded subsets into pre-compact sets. We divide the proof into four steps:

Step 1. For each bounded subset $D \subset P$, prove that $\left\{A_{x}: x \in \bar{D}\right\}$ is bounded in $R$. 
Denote

$$
L_{1}=\sup \left\{\max _{n \in N_{0}} \frac{|x(n)|}{1+\sum_{s=0}^{n-1} \frac{1}{\phi^{-1}(p(s))}}, \sup _{n \in N_{0}} \phi^{-1}(p(n))|\Delta x(n)|: x \in \bar{D}\right\}
$$

and

$$
f_{L_{1}}(j)=\max _{|x|,|y| \leq L_{1}}\left|f\left(j,\left(1+\sum_{s=0}^{j-1} \frac{1}{\phi^{-1}(p(s))}\right) x, \frac{y}{\phi^{-1}(p(j))}\right)\right| .
$$

Since $f$ is a Caratheodory function, it follows from 2.11 that

$$
0 \leq A_{x} \leq \delta \sum_{j=0}^{\infty} f_{L_{1}}(j)<\infty
$$

Hence $\left\{A_{x}: x \in \bar{D}\right\}$ is bounded in $R$.

Step 2. For each bounded subset $D \subset P$, and each $x_{0} \in D$, prove that $T$ is continuous at $x_{0}$.

For $x_{0} \in D$ and $x_{n} \in D$ with $x_{n} \rightarrow x_{0}(n \rightarrow+\infty)$ in $D$.

Denote $u_{n}(k)=\left(T x_{n}\right)(k), u_{0}(k)=\left(T x_{0}\right)(k)$ for all $k \in N_{0}$. We prove that $T$ is continuous at $x_{0}$, i.e., $u_{n} \rightarrow u_{0}(n \rightarrow+\infty)$. Let $A_{x_{0}}$ be defined by

$$
\begin{gathered}
=\frac{\sum_{n=1}^{\infty} \beta_{n}}{1-\sum_{n=1}^{\infty} \alpha_{n}} \sum_{n=1}^{\infty} \alpha_{n} \sum_{j=0}^{n-1} \phi^{-1}\left(\frac{A_{x_{0}}+\sum_{s=j}^{\infty} f\left(s, x_{0}(s), \Delta x_{0}(s)\right)}{p(j)}\right) \\
+\sum_{n=1}^{\infty} \beta_{n} \sum_{j=0}^{n-1} \phi^{-1}\left(\frac{A_{x_{0}}+\sum_{s=j}^{\infty} f\left(s, x_{0}(s), \Delta x_{0}(s)\right)}{p(j)}\right) .
\end{gathered}
$$

First, we prove that $A_{x}$ are continuous in $x$, i.e.,

$$
A_{x_{n}} \rightarrow A_{x_{0}}, n \rightarrow+\infty
$$

It follows from Step 1 that $A_{x_{n}}$ is bounded. Without loss of generality, suppose that $A_{x_{n}} \rightarrow \bar{A} \neq A_{x_{0}}$.

It is easy to see that

$$
\begin{gathered}
\lim _{n \rightarrow+\infty}\left[\frac{1}{1-\sum_{n=1}^{\infty} \alpha_{n}} \sum_{n=1}^{\infty} \alpha_{n} \sum_{j=0}^{n-1} \phi^{-1}\left(\frac{\left.A_{x_{n}}+\sum_{s=j}^{\infty} f\left(s, x_{n}(s), \Delta x_{n}(s)\right)\right)}{p(j)}\right)\right. \\
\left.+\sum_{j=0}^{n-1} \phi^{-1}\left(\frac{\left.A_{x_{n}}+\sum_{s=j}^{\infty} f\left(s, x_{n}(s), \Delta x_{n}(s)\right)\right)}{p(j)}\right)\right]
\end{gathered}
$$




$$
\begin{gathered}
=\frac{1}{1-\sum_{n=1}^{\infty} \alpha_{n}} \sum_{n=1}^{\infty} \alpha_{n} \sum_{j=0}^{n-1} \phi^{-1}\left(\frac{\left.\bar{A}+\lim _{n \rightarrow+\infty} \sum_{s=j}^{\infty} f\left(s, x_{n}(s), \Delta x_{n}(s)\right)\right)}{p(j)}\right) \\
+\sum_{j=0}^{n-1} \phi^{-1}\left(\frac{\left.\bar{A}+\lim _{n \rightarrow+\infty} \sum_{s=j}^{\infty} f\left(s, x_{n}(s), \Delta x_{n}(s)\right)\right)}{p(j)}\right) \\
=\frac{1}{1-\sum_{n=1}^{\infty} \alpha_{n}} \sum_{n=1}^{\infty} \alpha_{n} \sum_{j=0}^{n-1} \phi^{-1}\left(\frac{\left.\bar{A}+\sum_{s=j}^{\infty} f\left(s, x_{0}(s), \Delta x_{0}(s)\right)\right)}{p(j)}\right) \\
+\sum_{j=0}^{n-1} \phi^{-1}\left(\frac{\left.\bar{A}+\sum_{s=j}^{\infty} f\left(s, x_{0}(s), \Delta x_{0}(s)\right)\right)}{p(j)}\right) \\
=\bar{u}(k) .
\end{gathered}
$$

One sees that $\bar{A}=\lim _{n \rightarrow \infty} \phi^{-1}(p(n)) \Delta \bar{u}(n)$ and $\bar{u}$ satisfies

$$
\bar{u}(0)-\sum_{n=1}^{\infty} \alpha_{n} \bar{u}(n)=0, \lim _{n \rightarrow \infty} \frac{\bar{u}(n)}{1+\sum_{s=0}^{n-1} \frac{1}{\phi^{-1}(p(s))}}-\sum_{i=1}^{\infty} \beta_{i} \bar{u}(i)=0 .
$$

So

$$
\begin{aligned}
\phi^{-1}(\bar{A})= & \frac{\sum_{n=1}^{\infty} \beta_{n}}{1-\sum_{n=1}^{\infty} \alpha_{n}} \sum_{n=1}^{\infty} \alpha_{n} \sum_{j=0}^{n-1} \phi^{-1}\left(\frac{\bar{A}+\sum_{s=j}^{\infty} f\left(s, x_{0}(s), \Delta x_{0}(s)\right)}{p(j)}\right) \\
& +\sum_{n=1}^{\infty} \beta_{n} \sum_{j=0}^{n-1} \phi^{-1}\left(\frac{\bar{A}+\sum_{s=j}^{\infty} f\left(s, x_{0}(s), \Delta x_{0}(s)\right)}{p(j)}\right) .
\end{aligned}
$$

It follows from Lemma 2 that $\bar{A}=A_{x_{0}}$. Hence

$$
A_{x_{n}} \rightarrow \bar{A}=A_{x_{0}}, n \rightarrow+\infty .
$$

This together with the continuous property of $f$ implies that $T$ is continuous at $x_{0}$.

Step 3. For each bounded subset $\Omega \subset P$, prove that $T \Omega$ is bounded.

In fact, for each bounded subset $\Omega \subseteq D$, and $x \in \Omega$. Suppose

$$
\|x\|=\max \left\{\sup _{n \in N_{0}} \frac{|x(n)|}{1+\sum_{s=0}^{n-1} \frac{1}{\phi^{-1}(p(s))}}, \max _{n \in[0, N+1]} \phi^{-1}(p(n))|\Delta x(n)|\right\} \leq M_{1}
$$

and Step 1 implies that there exist constants $M_{2}>0$ such that $\left|A_{x}\right|<M_{2}$ for all $x \in \Omega$. Then

$$
\frac{|(T x)(n)|}{1+\sum_{s=0}^{n-1} \frac{1}{\phi^{-1}(p(s))}}
$$




$$
\begin{aligned}
& =\frac{1}{1+\sum_{s=0}^{n-1} \frac{1}{\phi^{-1}(p(s))}} \\
& \left(\frac{1}{1-\sum_{n=1}^{\infty} \alpha_{n}} \sum_{n=1}^{\infty} \alpha_{n} \sum_{j=0}^{n-1} \phi^{-1}\left(\frac{A_{x}+\sum_{s=j}^{\infty} f(s, x(s), \Delta x(s))}{p(j)}\right)\right. \\
& \left.+\sum_{j=0}^{n-1} \phi^{-1}\left(\frac{A_{x}+\sum_{s=j}^{\infty} f(s, x(s), \Delta x(s))}{p(j)}\right)\right) \\
& \leq \frac{1}{1+\sum_{s=0}^{n-1} \frac{1}{\phi^{-1}(p(s))}} \\
& \left(\frac{1}{1-\sum_{n=1}^{\infty} \alpha_{n}} \sum_{n=1}^{\infty} \alpha_{n} \sum_{j=0}^{n-1} \phi^{-1}\left(\frac{M_{2}+\sum_{s=j}^{\infty} f(s, x(s), \Delta x(s))}{p(j)}\right)\right. \\
& \left.+\sum_{j=0}^{n-1} \phi^{-1}\left(\frac{M_{2}+\sum_{s=j}^{\infty} f(s, x(s), \Delta x(s))}{p(j)}\right)\right) \\
& \leq \phi^{-1}\left(M_{2}+\sum_{s=0}^{\infty} f_{M_{1}}(s)\right) \\
& \frac{\frac{1}{1-\sum_{n=1}^{\infty} \alpha_{n}} \sum_{n=1}^{\infty} \alpha_{n} \sum_{j=0}^{n-1} \phi^{-1}\left(\frac{1}{p(j)}\right)+\sum_{j=0}^{n-1} \phi^{-1}\left(\frac{1}{p(j)}\right)}{1+\sum_{s=0}^{n-1} \frac{1}{\phi^{-1}(p(s))}} \\
& \leq \phi^{-1}\left(M_{2}+\sum_{s=0}^{\infty} f_{M_{1}}(s)\right) \\
& \sup _{n \in N_{0}} \frac{\frac{1}{1-\sum_{n=1}^{\infty} \alpha_{n}} \sum_{n=1}^{\infty} \alpha_{n} \sum_{j=0}^{n-1} \phi^{-1}\left(\frac{1}{p(j)}\right)+\sum_{j=0}^{n-1} \phi^{-1}\left(\frac{1}{p(j)}\right)}{1+\sum_{s=0}^{n-1} \frac{1}{\phi^{-1}(p(s))}} \\
& =: M_{3} \text {, }
\end{aligned}
$$

where $f_{M_{1}}(j)=\max _{|x| \leq M_{1},|y| \leq M_{1} \leq M_{1}}\left|f\left(j,\left(1+\sum_{s=0}^{j-1} \frac{1}{\phi^{-1}(p(s))}\right) x, \frac{y}{\phi^{-1}(p(j))}\right)\right|$. Similarly, one has that

$$
\phi^{-1}(p(n))|\Delta(T x)(n)|=\left|\phi^{-1}\left(A_{x}+\sum_{j=n}^{\infty} f(j, x(j), \Delta x(j))\right)\right|
$$




$$
\leq \phi^{-1}\left(M_{2}+\sum_{j=0}^{\infty} f_{M_{1}}(j)\right)=: M_{4} .
$$

It follows that $T \Omega$ is bounded.

Step 4. For each bounded subset $\Omega \subset P$, prove that $T \Omega$ is pre-compact.

Similarly to Step 3, we see $\left|A_{x}\right| \leq M_{2}$, we get

$$
A_{x}+\sum_{n=0}^{\infty} f(n, x(n), \Delta x(n)) \leq \phi\left(M_{4}\right) .
$$

Note $\phi^{-1}(x)=|x|^{q-2} x$. Then there $\xi \in\left[A_{x}, A_{x}+\sum_{j=n}^{\infty} f(j, x(j), \Delta x(j))\right]$ such that

$$
\begin{gathered}
\left|\phi^{-1}(p(n)) \Delta(T x)(n)-\phi^{-1}\left(A_{x}\right)\right| \\
=\left|\phi^{-1}\left(A_{x}+\sum_{j=n}^{\infty} f(j, x(j), \Delta x(j))\right)-\phi^{-1}\left(A_{x}\right)\right| \\
=(q-1) \xi^{q-2} \sum_{j=n}^{\infty} f(j, x(j), \Delta x(j)) \\
\leq(q-1) \phi\left(M_{4}\right)^{q-2} \sum_{j=n}^{\infty} f(j, x(j), \Delta x(j)) \\
\leq(q-1) \phi\left(M_{4}\right)^{q-2} \sum_{j=n}^{\infty} f_{M_{1}}(j) \\
\rightarrow 0 \text { uniformly as } n \rightarrow \infty .
\end{gathered}
$$

For any $\epsilon>0$, there exists $N_{1, \epsilon}>0$ such that

$$
\left|\phi^{-1}\left(p\left(n_{1}\right)\right) \Delta(T x)\left(n_{1}\right)-\phi^{-1}\left(p\left(n_{2}\right)\right) \Delta(T x)\left(n_{2}\right)\right|<\epsilon, n>N_{1, \epsilon} .
$$

Since $\lim _{n \rightarrow \infty} \phi^{-1}(p(n)) \Delta(T x)(n)=\phi^{-1}\left(A_{x}\right)$ uniformly, we know from the same methods used in the proof of Lemma 2 that

$$
\lim _{n \rightarrow \infty} \frac{|(T x)(n)|}{1+\sum_{s=0}^{n-1} \frac{1}{\phi^{-1}(p(s))}}=\phi^{-1}\left(A_{x}\right) \text { uniformly. }
$$

So there exists $N_{2, \epsilon}>0$ such that

$$
\left|\frac{(T x)\left(n_{1}\right)}{1+\sum_{s=0}^{n_{1}-1} \frac{1}{\phi^{-1}(p(s))}}-\frac{(T x)\left(n_{2}\right)}{1+\sum_{s=0}^{n_{2}-1} \frac{1}{\phi^{-1}(p(s))}}\right|<\epsilon, n>N_{2, \epsilon} .
$$


Choose $N_{\epsilon}=\max \left\{N_{1, \epsilon}, N_{2, \epsilon}\right\}$. Then

$$
\mid \phi^{-1}\left(p\left(n_{1}\right) \Delta(T x)\left(n_{1}\right)-\phi^{-1}\left(p\left(n_{2}\right) \Delta(T x)\left(n_{2}\right) \mid<\epsilon,\right.\right.
$$

and

$$
\left|\frac{(T x)\left(n_{1}\right)}{1+\sum_{s=0}^{n_{1}-1} \frac{1}{\phi^{-1}(p(s))}}-\frac{(T x)\left(n_{2}\right)}{1+\sum_{s=0}^{n_{2}-1} \frac{1}{\phi^{-1}(p(s))}}\right|<\epsilon, n>N_{\epsilon} .
$$

One knows that $T \Omega$ is pre-compact. Lemma 5 with Steps 1, 2, 3 and 4 imply that $T$ is completely continuous.

Theorem 1. Choose $k_{1}, k_{2} \in N$ with $k_{1}<k_{2}$. Let $\mu$ be defined by $2.1, \delta$ by 2.3 and $M$ by 2.9. Suppose that there exist positive constants $e_{1}, e_{2}, c$ such that

$$
c \geq \frac{e_{2}}{\mu}>e_{2}>e_{1}>0 .
$$

Let

$$
\begin{aligned}
& Q=\phi\left(\frac{c}{M}\right) \frac{1}{1+\delta} ; \\
& W=\phi\left(\frac{e_{2}\left(1+\sum_{s=0}^{k_{2}-1} \frac{1}{\phi^{-1}(p(s))}\right)}{\sum_{j=0}^{k_{1}-1} \phi^{-1}\left(\frac{\left.\sum_{s=k_{1}}^{k_{2}} \frac{1}{2^{s+1}}\right)}{p(j)}\right)}\right) ; \\
& E=\frac{1}{1+\delta} \phi\left(\frac{e_{1}}{\left.\sup _{n \in N_{0}} \frac{\frac{1}{1-\sum_{n=1}^{\infty} \alpha_{n}} \sum_{n=1}^{\infty} \alpha_{n} \sum_{j=0}^{n-1} \phi^{-1}\left(\frac{1}{p(j)}\right)+\sum_{j=0}^{n-1} \phi^{-1}\left(\frac{1}{p(j)}\right)}{1+\sum_{s=0}^{n-1} \frac{1}{\phi^{-1}(p(s))}}\right) .} .\right.
\end{aligned}
$$

If $Q>W$ and

(A1) $f\left(n,\left(1+\sum_{s=0}^{n-1} \frac{1}{\phi^{-1}(p(s))}\right) u, \frac{v}{\phi^{-1}(p(n))}\right) \leq \frac{Q}{2^{n+1}}$ for all $n \in N_{0}, u \in[0, c], v \in$ $[0, c]$;

(A2) $f\left(n,\left(1+\sum_{s=0}^{n-1} \frac{1}{\phi^{-1}(p(s))}\right) u, \frac{v}{\phi^{-1}(p(n))}\right) \geq \frac{W}{2^{n+1}}$ for all $n \in\left[k_{1}, k_{2}\right], u \in$ $\left[e_{2}, \frac{e_{2}}{\mu}\right], v \in[0, c]$;

(A3) $f\left(n,\left(1+\sum_{s=0}^{n-1} \frac{1}{\phi^{-1}(p(s))}\right) u, \frac{v}{\phi^{-1}(p(n))}\right) \leq \frac{E}{2^{n+1}}$ for all $n \in N_{0}, u \in\left[0, e_{1}\right], v \in$ $[0, c]$;

then $B V P(3)$ has at least three positive solutions $x_{1}, x_{2}, x_{3}$ such that

$$
\sup _{n \in N_{0}} \frac{x_{1}(n)}{1+\sum_{s=0}^{n-1} \frac{1}{\phi^{-1}(p(s))}}<e_{1}, \min _{n \in\left[k_{1}, k_{2}\right]} \frac{x_{2}(n)}{1+\sum_{s=0}^{n-1} \frac{1}{\phi^{-1}(p(s))}}>e_{2},
$$


and

$$
\sup _{n \in N_{0}} \frac{x_{3}(n)}{1+\sum_{s=0}^{n-1} \frac{1}{\phi^{-1}(p(s))}}>e_{1}, \min _{n \in\left[k_{1}, k_{2}\right]} \frac{x_{3}(n)}{1+\sum_{s=0}^{n-1} \frac{1}{\phi^{-1}(p(s))}}<e_{2} .
$$

Proof. Let $X, P$ and $T$ be defined above. We complete the proof by using Lemma 1. By the definitions, it is easy to see that $\alpha, \psi$ are two nonnegative continuous concave functionals on the cone $P, \gamma, \beta, \theta$ are three nonnegative continuous convex functionals on the cone $P$.

One sees $\alpha(x) \leq \beta(x)$ for all $x \in P$. From Lemma 4, we have $\|x\| \leq M \gamma(x)$ for all $x \in P$.

Lemma 6 implies that $x=x(n)$ is a solution of BVP(3) if and only if $x$ is a solution of the operator equation $x=T x$ and $T: P \rightarrow P$ is completely continuous.

Corresponding to Lemma 1 , choose

$$
h=\mu e_{1}, d=e_{1}, a=e_{2}, b=\frac{e_{2}}{\mu}, c=c .
$$

Now, we prove that all conditions of Lemma 1 hold. One sees that $0<d<a$. The remainder is divided into five steps.

Step 1. Prove that $T: \overline{P_{c}} \rightarrow \overline{P_{c}}$;

For $x \in \overline{P_{c}}$, we have $\|x\| \leq c$. Then

$$
\begin{aligned}
& 0 \leq \frac{x(n)}{1+\sum_{s=0}^{n-1} \frac{1}{\phi^{-1}(p(s))}} \leq c, n \in N_{0}, \\
& 0 \leq \phi^{-1}(p(n)) \Delta x(n) \leq c \text { for } n \in N_{0} .
\end{aligned}
$$

So (A1) implies that

$$
\begin{gathered}
=f\left(n,\left(1+\sum_{s=0}^{n-1} \frac{1}{\phi^{-1}(p(s))}\right) \frac{x(n, x(n), \Delta x(n))}{1+\sum_{s=0}^{n-1} \frac{1}{\phi^{-1}(p(s))}}, \frac{1}{\phi^{-1}(p(n))} \phi^{-1}(p(n)) \Delta x(n)\right) \\
\leq \frac{Q}{2^{n+1}}, n \in N_{0} .
\end{gathered}
$$

It follows from Lemma 6 that $T x \in P$. One sees from Lemma 1 that

$$
0 \leq A_{x} \leq \delta \sum_{j=0}^{\infty} f(j, x(j), \Delta x(j))
$$

We have that

$$
\phi^{-1}(p(n))|\Delta(T x)(n)|=\left|\phi^{-1}\left(A_{x}+\sum_{j=n}^{\infty} f(j, x(j), \Delta x(j))\right)\right|
$$




$$
\begin{aligned}
& \leq \phi^{-1}\left((1+\delta) \sum_{j=0}^{\infty} f(j, x(j), \Delta x(j))\right) \\
& \leq \phi^{-1}\left((1+\delta) \sum_{j=0}^{\infty} \frac{Q}{2^{j+1}}\right) \\
& \leq \phi^{-1}(Q(1+\delta)) \leq c .
\end{aligned}
$$

From Lemma 5, we have

$$
\begin{aligned}
\frac{\mid(T x)(n)}{1+\sum_{s=0}^{n-1} \frac{1}{\phi^{-1}(p(s))}} & \leq M \sup _{n \in N_{0}} \phi^{-1}(p(n))|\Delta(T x)(n)| \\
& \leq M \phi^{-1}((1+\delta) Q) \leq c .
\end{aligned}
$$

It follows that

$$
\|T x\|=\max \left\{\max _{n \in N_{0}} \frac{|(T x)(n)|}{1+\sum_{s=0}^{n-1} \frac{1}{\phi^{-1}(p(s))}}, \max _{n \in N_{0}} \phi^{-1}(p(n))|\Delta(T x)(n)|\right\} \leq c .
$$

Then $T: \overline{P_{c}} \rightarrow \overline{P_{c}}$.

Step 2. Prove that

$$
\begin{gathered}
\{y \in P(\gamma, \theta, \alpha ; a, b, c) \mid \alpha(x)>a\} \\
=\left\{y \in P\left(\gamma, \theta, \alpha ; e_{2}, \frac{e_{2}}{\mu}, c\right) \mid \alpha(x)>e_{2}\right\} \neq \varnothing
\end{gathered}
$$

and $\alpha(T x)>e_{2}$ for every $x \in P\left(\gamma, \theta, \alpha ; e_{2}, \frac{e_{2}}{\mu}, c\right)$;

By the definition of $\mu$, we can choose $A, B$ such that

$$
\begin{gathered}
A \in\left[\left(1+\sum_{s=0}^{k_{2}-1} \frac{1}{\phi^{-1}(p(s))}\right) e_{2},\left(1+\sum_{s=0}^{k_{1}-1} \frac{1}{\phi^{-1}(p(s))}\right) \frac{e_{2}}{\mu}\right], \\
B \leq \min \left\{\frac{e_{2}}{\mu}, \frac{A}{\mu\left(1+\sum_{s=0}^{k_{2}-1} \frac{1}{\phi^{-1}(p(s))}\right)}\right\}
\end{gathered}
$$

and

$$
|A-B|<\frac{c}{\max \left\{\phi^{-1}\left(p\left(k_{1}-1\right)\right), \phi^{-1}\left(p\left(k_{2}\right)\right)\right\}} .
$$

Let

$$
x(n)=\left\{\begin{array}{l}
A, n \in\left[k_{1}, k_{2}\right] \\
B, n \notin\left[k_{1}, k_{2}\right]
\end{array}\right.
$$


Then $x \in P$ and

$$
\begin{gathered}
\alpha(x)=\min _{n \in\left[k_{1}, k_{2}\right]} \frac{x(n)}{1+\sum_{s=0}^{n-1} \frac{1}{\phi^{-1}(p(s))}} \geq \frac{A}{\left(1+\sum_{s=0}^{k_{2}-1} \frac{1}{\phi^{-1}(p(s))}\right)}>e_{2}, \\
\theta(x)=\sup _{n \in N_{0}} \frac{x(n)}{1+\sum_{s=0}^{n-1} \frac{1}{\phi^{-1}(p(s))}} \\
\leq \max \left\{B, \frac{A}{\left(1+\sum_{s=0}^{k_{1}-1} \frac{1}{\phi^{-1}(p(s))}\right)}, \frac{B}{\left(1+\sum_{s=0}^{k_{2}-1} \frac{1}{\phi^{-1}(p(s))}\right)}\right\} \leq \frac{e_{2}}{\mu}=b,
\end{gathered}
$$

and

$\gamma(x)=\sup _{n \in N_{0}} \phi^{-1}(p(n))|\Delta x(n)|=\max \left\{\phi^{-1}\left(p\left(k_{1}-1\right)\right), \phi^{-1}\left(p\left(k_{2}\right)\right)\right\}|A-B|<c$.

It follows that $\{y \in P(\gamma, \theta, \alpha ; a, b, c) \mid \alpha(x)>a\} \neq \varnothing$.

For $x \in P(\gamma, \theta, \alpha ; a, b, c)$, one has that

$$
\begin{gathered}
\alpha(x)=\min _{n \in\left[k_{1}, k_{2}\right]} \frac{x(n)}{1+\sum_{s=0}^{n-1} \frac{1}{\phi^{-1}(p(s))}} \geq e_{2}, \\
\theta(x)=\sup _{n \in N_{0}} \frac{x(n)}{1+\sum_{s=0}^{n-1} \frac{1}{\phi^{-1}(p(s))}} \leq \frac{e_{2}}{\mu},
\end{gathered}
$$

and

$$
\gamma(x)=\sup _{n \in N_{0}} \phi^{-1}(p(n))|\Delta x(n)| \leq c .
$$

Then

$$
e_{2} \leq \frac{x(n)}{1+\sum_{s=0}^{n-1} \frac{1}{\phi^{-1}(p(s))}} \leq \frac{e_{2}}{\mu}, n \in\left[k_{1}, k_{2}\right], \quad 0 \leq \phi^{-1}(p(n)) \Delta x(n) \leq c .
$$

Thus (A2) implies that

$$
f(n, x(n), \Delta x(n)) \geq \frac{W}{2^{n+1}}, n \in\left[k_{1}, k_{2}\right] .
$$

We get

$$
\begin{array}{r}
\alpha(T x)=\min _{n \in\left[k_{1}, k_{2}\right]} \frac{(T x)(n)}{1+\sum_{s=0}^{n-1} \frac{1}{\phi^{-1}(p(s))}} \\
>\frac{(T x)\left(k_{1}\right)}{1+\sum_{s=0}^{k_{2}-1} \frac{1}{\phi^{-1}(p(s))}} \\
=\frac{1}{1+\sum_{s=0}^{k_{2}-1} \frac{1}{\phi^{-1}(p(s))}}\left[\frac{1}{1-\sum_{n=1}^{\infty} \alpha_{n}}\right.
\end{array}
$$




$$
\begin{gathered}
\sum_{n=1}^{\infty} \alpha_{n} \sum_{j=0}^{n-1} \phi^{-1}\left(\frac{A_{x}+\sum_{s=j}^{\infty} f(s, x(s), \Delta x(s))}{p(j)}\right) \\
\left.+\sum_{j=0}^{k_{1}-1} \phi^{-1}\left(\frac{A_{x}+\sum_{s=j}^{\infty} f(s, x(s), \Delta x(s))}{p(j)}\right)\right] \\
\geq \frac{1}{1+\sum_{s=0}^{k_{2}-1} \frac{1}{\phi^{-1}(p(s))}} \sum_{j=0}^{k_{1}-1} \phi^{-1}\left(\frac{\sum_{s=j}^{\infty} f(s, x(s), \Delta x(s))}{p(j)}\right) \\
\geq \frac{1}{1+\sum_{s=0}^{k_{2}-1} \frac{1}{\phi^{-1}(p(s))}} \sum_{j=0}^{k_{1}-1} \phi^{-1}\left(\frac{\sum_{s=k_{1}}^{k_{2}} f(s, x(s), \Delta x(s))}{p(j)}\right) \\
\geq \frac{1}{1+\sum_{s=0}^{k_{2}-1} \frac{1}{\phi^{-1}(p(s))}} \sum_{j=0}^{k_{1}-1} \phi^{-1}\left(\frac{\sum_{s=k_{1}}^{k_{2}} \frac{W}{2^{s+1}}}{p(j)}\right)
\end{gathered}
$$

This completes Step 2.

Step 3. Prove that

$\{y \in Q(\gamma, \theta, \psi ; h, d, c) \mid \beta(x)<d\}=\left\{y \in Q\left(\gamma, \theta, \psi ; \mu e_{1}, e_{1}, c\right) \mid \beta(x)<e_{1}\right\} \neq \varnothing$ and

$$
\beta(T x)<e_{1} \text { for every } x \in Q(\gamma, \theta, \psi ; h, d, c)=Q\left(\gamma, \theta, \psi ; \mu e_{1}, e_{1}, c\right) ;
$$

Similarly to Step 2, we can see that $\{y \in Q(\gamma, \theta, \psi ; h, d, c) \mid \beta(x)<d\} \neq \varnothing$.

For $x \in Q(\gamma, \theta, \psi ; h, d, c)$, one has that

$$
\begin{aligned}
& \psi(x)=\min _{n \in\left[k_{1}, k_{2}\right]} \frac{x(n)}{1+\sum_{s=0}^{n-1} \frac{1}{\phi^{-1}(p(s))}} \geq \mu e_{1} \\
& \theta(x)=\sup _{n \in N_{0}} \frac{x(n)}{1+\sum_{s=0}^{n-1} \frac{1}{\phi^{-1}(p(s))}} \leq d=e_{1},
\end{aligned}
$$

and

$$
\gamma(x)=\sup _{n \in N_{0}} \phi^{-1}(p(n))|\Delta x(n)| \leq c .
$$

Hence we get that

$$
0 \leq \frac{x(n)}{1+\sum_{s=0}^{n-1} \frac{1}{\phi^{-1}(p(s))}} \leq e_{1}, n \in N_{0} ; 0 \leq \phi^{-1}(p(n)) \Delta x(n) \leq c, n \in N_{0} .
$$

Then (A3) implies that

$$
f(n, x(n), \Delta x(n)) \leq \frac{E}{2^{n+1}}, n \in N_{0} .
$$


So 2.16 implies that

$$
\begin{aligned}
& \beta(T x)=\sup _{n \in N_{0}} \frac{(T x)(n)}{1+\sum_{s=0}^{n-1} \frac{1}{\phi^{-1}(p(s))}} \\
& =\sup _{n \in N_{0}} \frac{1}{1+\sum_{s=0}^{n-1} \frac{1}{\phi^{-1}(p(s))}}\left[\frac{1}{1-\sum_{n=1}^{\infty} \alpha_{n}}\right. \\
& \sum_{n=1}^{\infty} \alpha_{n} \sum_{j=0}^{n-1} \phi^{-1}\left(\frac{A_{x}+\sum_{s=j}^{\infty} f(s, x(s), \Delta x(s))}{p(j)}\right) \\
& \left.+\sum_{j=0}^{n-1} \phi^{-1}\left(\frac{A_{x}+\sum_{s=j}^{\infty} f(s, x(s), \Delta x(s))}{p(j)}\right)\right] \\
& <\sup _{n \in N_{0}} \frac{\phi^{-1}\left((1+\delta) \sum_{s=0}^{\infty} f(s, x(s), \Delta x(s))\right)}{1+\sum_{s=0}^{n-1} \frac{1}{\phi^{-1}(p(s))}}\left[\frac{1}{1-\sum_{n=1}^{\infty} \alpha_{n}}\right. \\
& \left.\sum_{n=1}^{\infty} \alpha_{n} \sum_{j=0}^{n-1} \phi^{-1}\left(\frac{1}{p(j)}\right)+\sum_{j=0}^{n-1} \phi^{-1}\left(\frac{1}{p(j)}\right)\right] \\
& \leq \sup _{n \in N_{0}} \frac{\phi^{-1}\left((1+\delta) \sum_{s=0}^{\infty} \frac{E}{2^{j+1}}\right)}{1+\sum_{s=0}^{n-1} \frac{1}{\phi^{-1}(p(s))}}\left[\frac{1}{1-\sum_{n=1}^{\infty} \alpha_{n}} \sum_{n=1}^{\infty} \alpha_{n} \sum_{j=0}^{n-1} \phi^{-1}\left(\frac{1}{p(j)}\right)\right. \\
& \left.+\sum_{j=0}^{n-1} \phi^{-1}\left(\frac{1}{p(j)}\right)\right] \\
& \leq \phi^{-1}((1+\delta) E) \\
& \sup _{n \in N_{0}} \frac{\frac{1}{1-\sum_{n=1}^{\infty} \alpha_{n}} \sum_{n=1}^{\infty} \alpha_{n} \sum_{j=0}^{n-1} \phi^{-1}\left(\frac{1}{p(j)}\right)+\sum_{j=0}^{n-1} \phi^{-1}\left(\frac{1}{p(j)}\right)}{1+\sum_{s=0}^{n-1} \frac{1}{\phi^{-1}(p(s))}} \\
& \leq e_{1}=d \text {. }
\end{aligned}
$$

This completes Step 3.

Step 4. Prove that $\alpha(T y)>a$ for $y \in P(\gamma, \alpha ; a, c)$ with $\theta(T y)>b$;

For $x \in P(\gamma, \alpha ; a, c)=P\left(\gamma, \alpha ; e_{2}, c\right)$ with $\theta(T x)=\beta(T x)>b=\frac{e_{2}}{\mu}$, we have that

$$
\begin{aligned}
& \alpha(x)=\min _{n \in\left[k_{1}, k_{2}\right]} \frac{x(n)}{1+\sum_{s=0}^{n-1} \frac{1}{\phi^{-1}(p(s))}} \geq e_{2}, \\
& \gamma(x)=\sup _{n \in N_{0}} \phi^{-1}(p(n))|\Delta x(n)| \leq c,
\end{aligned}
$$




$$
\sup _{n \in N_{0}} \frac{(T x)(n)}{1+\sum_{s=0}^{n-1} \frac{1}{\phi^{-1}(p(s))}}>\frac{e_{2}}{\mu} .
$$

Then

$$
\alpha(T x)=\min _{n \in\left[k_{1}, k_{2}\right]} \frac{(T x)(n)}{1+\sum_{s=0}^{n-1} \frac{1}{\phi^{-1}(p(s))}} \geq \mu \beta(T x)>e_{2}=a .
$$

This completes Step 4.

Step 5. Prove that $\beta(T x)<d$ for each $x \in Q(\gamma, \beta ; d, c)$ with $\psi(T x)<h$.

For $x \in Q(\gamma, \beta ; d, c)$ with $\psi(T x)<h$, we have

$$
\begin{aligned}
\gamma(x) & =\sup _{n \in N_{0}} \phi^{-1}(p(n))|\Delta x(n)| \leq c, \\
\beta(x) & =\sup _{n \in N_{0}} \frac{x(n)}{1+\sum_{s=0}^{n-1} \frac{1}{\phi^{-1}(p(s))}} \leq d=e_{1}, \\
\psi(T x) & =\min _{n \in\left[k_{1}, k_{2}\right]} \frac{(T x)(n)}{1+\sum_{s=0}^{n-1} \frac{1}{\phi^{-1}(p(s))}}<h=\mu e_{1} .
\end{aligned}
$$

Then

$$
\beta(T x)=\sup _{n \in N_{0}} \frac{(T x)(n)}{1+\sum_{s=0}^{n-1} \frac{1}{\phi^{-1}(p(s))}} \leq \frac{1}{\mu} \min _{n \in\left[k_{1}, k_{2}\right]} \frac{(T x)(n)}{1+\sum_{s=0}^{n-1} \frac{1}{\phi^{-1}(p(s))}}<e_{1}=d .
$$

This completes the Step 5.

Then Lemma 1 implies that $T$ has at least three fixed points $y_{1}, y_{2}$ and $y_{3}$ such that

$$
\beta\left(y_{1}\right)<e_{1}, \alpha\left(y_{2}\right)>e_{2}, \beta\left(y_{3}\right)>e_{1}, \alpha\left(y_{3}\right)<e_{2} .
$$

Hence BVP(3) has three positive solutions $y_{1}, y_{2}$ and $y_{3}$ satisfying 2.14 and 2.15. The proof is complete.

\section{AN EXAMPLE}

In this section, we present an example to illustrate Theorem 1.

Example 1. Consider the following BVP

$$
\left\{\begin{array}{l}
\Delta^{2} x(n)=-f(n, x(n), \Delta x(n)), n \in N_{0} \\
x(0)=\sum_{n=1}^{\infty} \frac{1}{2^{n+1}} x(n) \\
\lim _{n \rightarrow \infty} \frac{x(n)}{1+n}=\sum_{n=1}^{\infty} \frac{1}{3^{n+1}} x(n)
\end{array}\right.
$$

where $f: N_{0} \times[0,+\infty)^{2} \rightarrow[0,+\infty)$ is a Caratheodory function.

Corresponding to $\operatorname{BVP}(3), p(n) \equiv 1, \alpha_{n}=\frac{1}{2^{n+1}}, \beta_{n}=\frac{1}{3^{n+1}}$ and $\phi(x)=x$. 
Choose the constant $k_{1}=10, k_{2}=10000, e_{1}=100, e_{2}=5400, c=3688400 \times$ $2^{10009}$. It is easy to see that

$$
\begin{aligned}
& \mu=\frac{1}{\max \left\{\phi^{-1}\left(p\left(k_{1}-1\right)\right), 1\right\}\left(1+\sum_{s=0}^{k_{2}-1} \frac{1}{\phi^{-1}(p(s))}\right)}=\frac{1}{10001}, \\
& M=\max \left\{1, \sup _{n \in N_{0}} \frac{\sum_{i=0}^{n-1} \frac{1}{\phi^{-1}(p(i))}+\frac{\sum_{n \in N} \alpha_{n} \sum_{i=0}^{n-1} \frac{1}{\phi^{-1}(p(i))}}{1-\sum_{i=1}^{\infty} \alpha_{i}}}{1+\sum_{i=0}^{n-1} \frac{1}{\phi^{-1}(p(i))}}\right\}=\frac{5}{4}, \\
& \delta \\
& =\frac{\phi\left(\frac{\sum_{n=1}^{\infty} \beta_{n}}{1-\sum_{n=1}^{\infty} \alpha_{n}} \sum_{n=1}^{\infty} \alpha_{n} \sum_{j=0}^{n-1} \phi^{-1}\left(\frac{1}{p(j)}\right)+\sum_{n=1}^{\infty} \beta_{n} \sum_{j=0}^{n-1} \phi^{-1}\left(\frac{1}{p(j)}\right)\right)}{1-\phi\left(\frac{\sum_{n=1}^{\infty} \beta_{n}}{1-\sum_{n=1}^{\infty} \alpha_{n}} \sum_{n=1}^{\infty} \alpha_{n} \sum_{j=0}^{n-1} \phi^{-1}\left(\frac{1}{p(j)}\right)+\sum_{n=1}^{\infty} \beta_{n} \sum_{j=0}^{n-1} \phi^{-1}\left(\frac{1}{p(j)}\right)\right)} \\
& =1 \text {, } \\
& Q=\phi\left(\frac{c}{M}\right) \frac{1}{1+\delta}=1498400 \times 2^{10009} ; \\
& W=\phi\left(\frac{e_{2}\left(1+\sum_{s=0}^{k_{2}-1} \frac{1}{\phi^{-1}(p(s))}\right)}{\phi^{-1}\left(\sum_{s=k_{1}}^{k_{2}} \frac{1}{2^{s+1}}\right) \sum_{j=0}^{k_{1}-1} \phi^{-1}\left(\frac{1}{p(j)}\right)}\right)=\frac{10001 \times 540 \times 2^{10009}}{2^{10000}-2^{9}} ; \\
& E=\frac{1}{1+\delta} \phi\left(\frac{e_{1}}{\sup _{n \in N_{0}} \frac{\frac{1}{1-\sum_{n=1}^{\infty} \alpha_{n}} \sum_{n=1}^{\infty} \alpha_{n} \sum_{j=0}^{n-1} \phi^{-1}\left(\frac{1}{p(j)}\right)+\sum_{j=0}^{n-1} \phi^{-1}\left(\frac{1}{p(j)}\right)}{1+\sum_{s=0}^{n-1} \frac{1}{\phi^{-1}(p(s))}}}\right)=40 .
\end{aligned}
$$

So $Q>W$ and $c>\frac{e_{2}}{\mu}>e_{2}>e_{1}>0$. If

$$
f_{0}(x)=\left\{\begin{array}{l}
20, x \in[0,100], \\
20+(x-100) \frac{\frac{1498400 \times 2^{10009}+\frac{10001 \times 540 \times 2^{10009}}{2}}{2}}{2^{10000}-2^{9}}-20 \\
\frac{15300-100}{490}-\frac{5}{343}(x-1554)+\frac{5}{343}, x \in[5400,5400 \times 10001], \\
\frac{1404-1554}{5400 \times 2^{10009}+\frac{10001 \times 540 \times 210009}{2^{10000}-2^{9}}}, \quad x \in[5400 \times 10001,+\infty),
\end{array}\right.
$$

and

Then

$$
f(n, x, y)=\frac{f_{0}\left(\frac{x}{1+n}\right)}{2^{n+1}}+\frac{|y|}{343 \times 3688400} .
$$

It is easy to check that if

$$
f(n,(1+n) x, y)=\frac{f_{0}(x)}{2^{n+1}}+\frac{|y|}{343 \times 3688400} .
$$


(A1) $f(n,(1+n) u, v) \leq \frac{Q}{2^{n+1}}$ for all $n \in N_{0}, u \in[0, c], v \in[0, c]$;

(A2) $f(n,(1+n) u, v) \geq \frac{w}{2^{n+1}}$ for all $n \in\left[k_{1}, k_{2}\right], u \in\left[e_{2}, e_{2} \mu\right], v \in[0, c]$;

(A3) $f(n,(1+n) u, v) \leq \frac{E}{2^{n+1}}$ for all $n \in N_{0}, u \in\left[0, e_{1}\right], v \in[0, c]$;

then Theorem 1 implies that BVP(21) has at least three positive solutions such that

$$
\sup _{n \in N_{0}} \frac{x_{1}(n)}{1+n}<100, \min _{n \in[10,10000]} \frac{x_{2}(n)}{1+n}>5400,
$$

and

$$
\sup _{n \in N_{0}} \frac{x_{3}(n)}{1+n}>100, \min _{n \in[10,10000]} \frac{x_{3}(n)}{1+n}<5400 .
$$

Remark 1. BVP(21) in Example 1 can not be solved by the theorems in [2-4, 16, 17].

\section{REFERENCES}

[1] R. P. Agarwal, Difference equations and inequalities: theory, methods, and applications. 2nd, revised and expanded ed., ser. Pure and Applied Mathematics. New York: Marcel Dekker, 2000, vol. 228.

[2] R. P. Agarwal, M. Bohner, and D. O'Regan, "Time scale boundary value problems on infinite intervals," J. Comput. Appl. Math., vol. 141, no. 1-2, pp. 27-34, 2002.

[3] R. P. Agarwal and D. O'Regan, "Boundary value problems for general discrete systems on infinite intervals," Comput. Math. Appl., vol. 33, no. 7, pp. 85-99, 1997.

[4] R. P. Agarwal and D. O'Regan, "Discrete systems on infinite intervals," Comput. Math. Appl., vol. 35, no. 9, pp. 97-105, 1998.

[5] R. P. Agarwal and D. O'Regan, "Cone compression and expansion fixed point theorems in fréchet spaces with applications," J. Differ. Equations, vol. 171, no. 2, pp. 412-429, 2001.

[6] R. P. Agarwal and D. O'Regan, "Nonlinear Urysohn discrete equations on the infinite interval:a fixed-point approach," Comput. Math. Appl., vol. 42, no. 3-5, pp. 273-281, 2001.

[7] R. I. Avery, "A generalization of the Leggett-Williams fixed point theorem," Math. Sci. Res. HotLine, vol. 3, no. 7, pp. 9-14, 1999.

[8] R. I. Avery and A. C. Peterson, "Three positive fixed points of nonlinear operators on ordered Banach spaces," Comput. Math. Appl., vol. 42, no. 3-5, pp. 313-322, 2001.

[9] W.-S. Cheung, J. Ren, P. J. Wong, and D. Zhao, "Multiple positive solutions for discrete nonlocal boundary value problems," J. Math. Anal. Appl., vol. 330, no. 2, pp. 900-915, 2007.

[10] J. R. Graef, L. Kong, and B. Yang, "Positive solutions for third order multi-point singular boundary value problems," Czech. Math. J., vol. 60, no. 1, pp. 173-182, 2010.

[11] Y. Li and L. Lu, "Existence of positive solutions of $p$-Laplacian difference equations," Appl. Math. Lett., vol. 19, no. 10, pp. 1019-1023, 2006.

[12] Y. Liu, "Positive solutions of multi-point BVPs for second-order $p$-Laplacian difference equations," Commun. Math. Anal., vol. 4, no. 1, pp. 58-77, 2008.

[13] Y. Liu and W. Ge, "Twin positive solutions of boundary value problems for finite difference equations with $p$-Laplacian operator," J. Math. Anal. Appl., vol. 278, no. 2, pp. 551-561, 2003.

[14] R. Ma and Y. N. Raffoul, "Positive solutions of three-point nonlinear discrete second order boundary value problem," J. Difference Equ. Appl., vol. 10, no. 2, pp. 129-138, 2004.

[15] H. Pang, H. Feng, and W. Ge, "Multiple positive solutions of quasi-linear boundary value problems for finite difference equations," Appl. Math. Comput., vol. 197, no. 1, pp. 451-456, 2008. 
[16] A. S. V. Ravi Kanth and Y. N. Reddy, "A numerical method for solving two-point boundary value problems over infinite intervals," Appl. Math. Comput., vol. 144, no. 2-3, pp. 483-494, 2003.

[17] Y. Tian and W. Ge, "Multiple positive solutions of boundary value problems for second-order discrete equations on the half-line," J. Difference Equ. Appl., vol. 12, no. 2, pp. 191-208, 2006.

[18] P. J. Y. Wong and L. Xie, "Three symmetric solutions of Lidstone boundary value problems for difference and partial difference equations," Comput. Math. Appl., vol. 46, no. 6-9, pp. 1445-1460, 2003.

[19] J. Yu and Z. Guo, "On generalized discrete boundary value problems of Emden-Fowler equation," Science in China (Ser. A Mathematics), vol. 36, no. 7, pp. 721-732, 2006.

Authors' addresses

\section{Jianye Xia}

Department of Applied Mathematics, Guangdong University of Finance, Guangzhou 510000, P.R.China E-mail address: liuyuji888@sohu.com

\section{Yuji Liu}

Department of Mathematics, Guangdong University of Business Studies Guangzhou 510000, P.R.China 